\title{
Converging technologies to enable induced pluripotent stem cells in drug discovery
} "Induced pluripotent stem cell research has proceeded at an incredible pace ... The
identification of robust differentiation protocols that result in homogeneous and
stable cell populations for therapeutically relevant lineages is the next milestone."

Induced pluripotent stem (iPS) cells are similar to embryonic stem cells (ESCs) in their potential to differentiate into many cell types. The significant advantage of iPS cells is the ability to create pluripotent lines from any genetic background, including disease states. The ability to efficiently create and differentiate iPS cells to reproducibly recapitulate human physiology will have a significant impact on disease research, drug discovery, toxicity testing and, ultimately, cell therapy.

Since its first demonstration, somatic cell reprogramming has evolved from a niche expertise performed by a few pioneering laboratories to a common technique used by researchers working in a multitude of fields [1]. While many methods of reprogramming have been developed, the use of integrating retroviral or lentiviral systems remains the most commonly used technique. However, to advance the commercial and clinical applications, some major questions in the field are:

- Are all iPS cells created equal and do they need to be?

- Does the reprogramming method matter?

- Does the starting cell-type make a difference for subsequent differentiation potential and stability?

With the continued advancement and indepth analysis of reprogramming, cell culture and differentiation methods, the answers to these questions are beginning to develop.

Induced pluripotent stem cell colonies are generally identified based on morphology and immunostaining of a few pluripotency markers. Characterization includes further immunofluorescence and expression analysis of key markers, determining the methylation status of endogenous pluripotency genes, the inactivation of exogenous genes and the potential to differentiate into the three germ layers via embroid body or teratoma analysis. Clearly, iPS cell lines generated using different systems (or the same viral systems that result in differing patterns of genomic integration) can perform with equal efficiency and effect in these standard assays. However, for both disease models and cell therapy, the true test of an iPS cell line is its potential to differentiate into specific lineages and maintain stable progenitor or postmitotic status.

Recent studies have demonstrated significant differences between iPS cell clones generated using the same viral systems, with a large percentage of clones forming tumors in rodents when transplanted as differentiated neurospheres [2]. The tumorigenic potential of clones was seen to be dependent on the starting cell type, suggesting that this may play a role in the 'quality' of an iPS cell clone and its differentiated product. However, there is a clear risk that the use of integrating viral systems for the generation of iPS cells may lead to insertional mutagenesis or exongenous transgene reactivation. One partial solution to this problem is the use of nuclease-directed recombination of factors to 'safe-harbor' sites of the genome. A more complete solution is to remove the virus or use nonintegrating, minimally invasive methods of reprogramming.

Both genetic and nongenetic technologies may fit the description of minimally invasive reprogramming, and methods using excisable cre/lox vector [3], piggyBac transposon system [4], adenoviral vectors [5], episomal plasmids [6] and cellpenetrating proteins [7] have all been described. Although it has yet to be determined which of these reprogramming methods is best for in vitro applications, prescreening for iPS cell-derived lineage stability would be a prerequisite for commercial drug screening efforts. Clearly, in vivo clinical applications of the technology would necessitate minimally invasive reprogramming

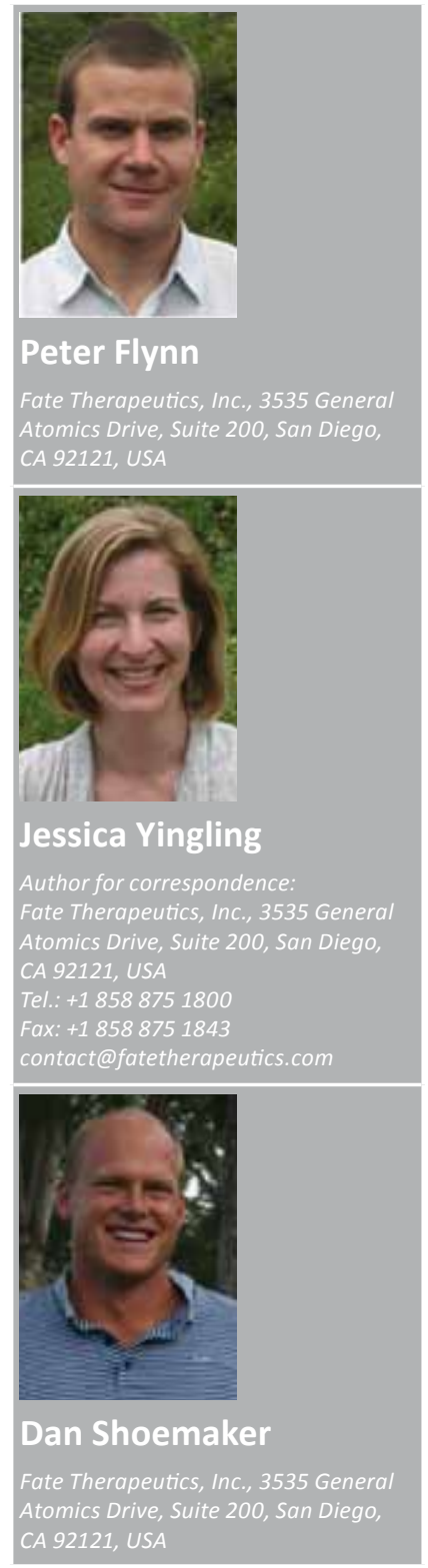


technologies, preferably protein and small molecules, and even more rigorous characterization of iPS cell-derived products.

Molecular characterization approaches have now evolved to include a comprehensive analysis of gene expression, protein levels and total genome epigenetic status. A detailed comparison of the various methods of reprogramming from the same starting cell type, as well as clone-toclone comparison within the same method would be informative for the field. Such a study should include the typical pluripotency characterization as well as global expression and epigenetic analysis followed by an assessment of differentiation potential, biological function and stability of specific lineages. Initial studies comparing the genome-wide expression of a number of iPS cell and ESC clones in both the pluripotent state and postdifferentiation to specific lineages demonstrate an overall similarity between the two cell types, with an equivalent distribution within the expression space for both iPS cells and ESCs [8]

\section{"The ability to efficiently create and differentiate iPS cells to reproducibly recapitulate human physiology will have a significant impact on disease research, drug discovery, toxicity testing and, ultimately, cell therapy."}

It is clear from studies using multiple ESC lines that some have a greater potential for differentiation to certain lineages. Whether iPS cells show the same differential or are more conformed in their differentiation is unclear. Recently iPS cells generated using lentiviruses were shown to exhibit decreased differentiation and expansion capability as well as early senescence when compared with ESC lines [9]. In other systems, excision of transgenes were seen to affect the differentiation potential of iPS cells [10]. A study using excisable lentiviral systems compared genome-wide expression of an ESC line with an iPS cell clone where the transgenes were either integrated into the genome or removed [3]. A total of 271 genes were significantly differentially expressed within the ESC line compared with iPS cells formed via viral integration, but this number dropped to 48 genes in the case of excised, factor-free iPS cells. Variation due to the use of different reprogramming methods and clonal differences for the most commonly used viral methods will require further exploration.

Small molecule inhibitors of specific signaling pathways have been used to great effect at multiple stages of iPS cell generation, maintenance and expansion as well as establishment of different levels of pluripotency. Combinations of small molecules have been used to reduce the number of transcription factors required for reprogramming, and, in a recent study, cocktails of molecules were seen to greatly improve virus reprogramming efficiency and support enzymatic passaging [11]. These, and other molecules, should improve inefficient genetic-free reprogramming approaches and enable reprogramming in reduced culture sizes. Additional important steps in the commercial application of both ESCs and iPS cells include the identification of small molecule additives to cell culture media to enable the generation of iPS cells in feederfree, defined environments while maintaining pluripotency and viability.

Small molecules have also demonstrated to be able to support reprogramming to a more naive state. Comparisons of mouse ESCs derived from either blastocyst inner cell mass or epiblast have demonstrated there to be different levels of pluripotency [12]. Human ESCs and conventional iPS cells are seen to be molecularly similar to mouse epiblast ESCs. The use of small molecule inhibitors of key pathways and the addition of leukemia inhibitory factor to the culture media was able to convert the human cells to a more naive state, equivalent to mouse inner cell mass ESCs [13]. In another recent study, comparison of genetically identical ESCs and iPS cells correlated with the inability of some iPS cell clones to contribute effectively to chimeras or generate iPS cell-derived animals with the aberrant silencing of a particular imprinted gene cluster [14]. This difference between iPS cells and ESCs could be overcome with the use of small molecule histone deacetylase inhibitors. It is therefore clear that continued research and use of small molecules will be vital for academic and commercial applications of the reprogramming process and iPS cell technology.

Quantitative biology tools are now being used to great effect in defining pluripotency and differentiation states. Techniques, such as genome-wide expression profiles, including gene and miRNA transcripts; epigenetic analysis and proteomic methods, such as antibodybased and mass spectrometry approaches, will all aid in the understanding and application of iPS cell technology. Identifying robust signatures for pluripotency in each of these technologies will be the next stage for the field. Analysis of multiple ESC lines will provide a baseline and expected deviation for each parameter, although variables such as starting 
cell type, method of reprogramming and culture environment may impact on molecular signatures for iPS cell clones. The power of iPS cell technology for commercial drug discovery lies in the potential to derive any cell lineage from any starting genetic background, including disease states. The challenge will be to generate models that exhibit physiologically relevant disease phenotypes in vitro. The development of robust and physiologically relevant differentiation protocols for pluripotent cells will also benefit from the increased use of molecular analytical tools. The isolation of desired terminally differentiated or progenitor cell types from tissue and their detailed analysis will again provide molecular signatures that can act as guides and target profiles for multiplex differentiation protocols.

Induced pluripotent stem cell research has proceeded at an incredible pace. The development of cell culture conditions, including small molecule additives to aid in the establishment, maintenance and expansion of pluripotent cells, has been a major advance to the field. The identification of robust differentiation protocols that result in homogeneous and stable cell populations for therapeutically relevant lineages is the next milestone. Comprehensive molecular and functional comparison of iPS cells generated using different starting cell types and methods will be vital for the optimization of reprogramming strategies. The implementation of locispecific markers and comparative expression and protein signatures seen in iPS cell-derived cell lineages with target cell types from the in vivo developmental process will be required to establish robust differentiation protocols. As the field strives to effectively use iPS cells to model disease phenotypes and derive relevant cell lineages for commercial and clinical use, the ability to converge quantitative molecular biology and proteomic analysis with reprogramming and differentiation technologies will support full maturation of this technology.

\section{Financial \& competing interests disclosure}

The authors are employees of Fate Therapeutics, Inc. The authors have no other relevant affiliations or financial involvement with any organization or entity with a financial interest in or financial conflict with the subject matter or materials discussed in the manuscript apart from those disclosed.

No writing assistance was utilized in the production of this manuscript.

\section{Bibliography}

1 Takahashi K, Yamanaka S: Induction of pluripotent stem cells from mouse embryonic and adult fibroblast cultures by defined factors. Cell 126, 663-676 (2006).

2 Miura K, Okada Y, Aoi T et al.: Variation in the safety of induced pluripotent stem cell lines. Nat. Biotech. 27(8), 743-745 (2009).

3 Soldner F, Hockemeyer D, Beard C et al.: Parkinson's disease patient-derived induced pluripotent stem cells free of viral reprogramming factors. Cell 136(5), 964-977 (2009).

4 Woltjen K, Michael IP, Mohseni P et al.: piggyBac transposition reprograms fibroblasts to induced pluripotent stem cells. Nature 458(7239), 766-770 (2009).

5 Stadtfeld M, Nagaya M, Utikal J, Weir G, Hochedlinger K: Induced pluripotent stem cells generated without viral integration. Science 322(5903), 945-949 (2008).
6

Okita K, Nakagawa M, Hyenjong H, Ichisaka T, Yamanaka S: Generation of mouse induced pluripotent stem cells without viral vectors. Science 322(5903), 949-953 (2008).

7 Zhou H, Wu S, Joo JY, Zhu S et al: Generation of induced pluripotent stem cells using recombinant proteins. Cell Stem Cell 4(5), 381-384 (2009).

8 Aiba K, Nedorezov T, Piao Y et al.: Defining developmental potency and cell lineage trajectories by expression profiling of differentiating mouse embryonic stem cells. DNA Res. 16(1), 73-80 (2009).

9 Feng Q, Lu SJ, Klimanskaya I et al.: Hemangioblastic derivatives from human induced pluripotent stem cells exhibit limited expansion and early senescence. Stem Cells 28(4), 704-712 (2010).

10 Sommer CA, Sommer AG, Longmire TA et al.: Excision of reprogramming transgenes improves the differentiation potential of iPS cells generated with a single excisable vector. Stem Cells 28(1), 64-74 (2010).

11 Lin T, Ambasudhan R, Yuan X et al.: A chemical platform for improved induction of human iPSCs. Nat. Meth. 6(11), 805-808 (2009).

12 Nichols J, Smith A: Naive and primed pluripotent states. Cell Stem Cell 4(6), 487-492 (2009).

13 Hanna J, Cheng AW, Saha K et al.: Human embryonic stem cells with biological and epigenetic characteristics similar to those of mouse ESCs. Proc. Natl Acad. Sci. USA 107(20), 9222-9227 (2010).

14 Stadtfeld M, Apostolou E, Akutsu H et al.: Aberrant silencing of imprinted genes on chromosome $12 \mathrm{qF} 1$ in mouse induced pluripotent stem cells. Nature 465(7295), 175-181 (2010). 